\title{
Diagnosis of Cirrhotic Portal Hypertension and Compensatory Circulation Using Transsplenic Portal Scintigraphy with 99m-Tc-Phytate
}

\author{
$\mathrm{Li} \mathrm{Gao}^{1}$, Fuyong Yang ${ }^{1}$, Changzheng Ren ${ }^{1}$, Jiankui $\mathrm{Han}^{1}$, Youan $\mathrm{Zhao}^{2}$, and ${\mathrm{Hua} \mathrm{Li}^{3}}^{3}$
}

${ }^{I}$ Department of Nuclear Medicine, Qilu Hospital of Shandong University, Jinan, China; ${ }^{2}$ Department of Digestive Internal Medicine, Qilu Hospital of Shandong University, Jinan, China; and ${ }^{3}$ Department of Digestive Internal Medicine, Second Hospital of Shandong University, Jinan, China

\begin{abstract}
Our objective was to investigate the diagnostic value and clinical significance of transsplenic portal scintigraphy in cirrhotic portal hypertension and compensatory circulation. Methods: Transsplenic portal scintigraphy, ultrasound, and gastroscopy were performed on 50 patients with cirrhotic portal hypertension and on 10 controls. According to the Child-Pugh classification, 15 patients with cirrhosis were Child A, 19 were Child B, and 16 were Child C. Results: In the control group, the splenoportal vein was shaped like the letter $S$, and the portosystemic shunt index was $0.19 \pm 0.07$. Portal hypertension portosystemic shunts were of 3 types: intrahepatic (13 patients; index, $0.52 \pm 0.19$ ), compensatory (31 patients; index, $0.64 \pm 0.28)$; and completely extrahepatic ( 6 patients; index, $0.91 \pm 0.03$ ). Collateral vessels were uphill, downhill, or complex. The portosystemic shunt index increased as cirrhosis and esophageal varices increased. There was statistical significance among groups $(P<0.05$ or $<0.01)$. Conclusion: Transsplenic portal scintigraphy was sensitive for detecting the number and location of shunts and will allow for improved surgical planning.
\end{abstract}

Key Words: cirrhosis; portal hypertension; portal scintigraphy; compensatory circulation; portosystemic shunt

J Nucl Med 2010; 51:52-56

DOI: 10.2967/jnumed.109.067983

\section{C}

irrhotic portal hypertension, which may lead to the formation of extensive and perplexing portosystemic compensatory circulation, is responsible for many complications such as hemorrhage resulting from rupture of esophageal and fundal gastric varices and hepatocerebral disease. The treatment of portal hypertension still remains formidable in clinical practice because of sophisticated pathogenetic conditions, risk factors, and operative difficulties. It is crucial for the clinician to know the anatomic

Received Jul. 6, 2009; revision accepted Sep. 8, 2009.

For correspondence or reprints contact: Jiankui Han, Department of Nuclear Medicine, Qilu Hospital of Shandong University, Jinan, 250012, China.

E-mail: jkhan@sdu.edu.cn

COPYRIGHT @ 2010 by the Society of Nuclear Medicine, Inc. structure of the portal system, the distribution, and the hemodynamic alterations of collateral vessels in order to choose the correct therapeutic regimen, predict bleeding and recurrence, improve the curative effect, and reduce treatment time $(1,2)$. There are advantages, as well as disadvantages, to the commonly used imaging methods for diagnosis of portal hypertension: CT, nuclear magnetic resonance, ultrasound, and per rectum portal scintigraphy.

Portography by multislice spiral CT and enhanced nuclear magnetic resonance, with the advantages of quick scanning, high spatial resolution, and powerful 3-dimensional postprocessing, can be used to acquire high-quality vein images that show blood vessels precisely but cannot demonstrate hemodynamic alterations of the portal vein (3-5). Ultrasound was formerly used to diagnose portal hypertension by determining the inner diameter of the portal vein, because portal hypertension results in circuitous and distended blood vessels. However, Kudo et al. (6) and Berzigotti et al. (7) reported that measurement of the inner diameter was insufficient to diagnose portal hypertension because of the lack of a significant correlation between diameter and portal vein pressure. Per rectum portal scintigraphy is used to determine portosystemic shunting by the heart-to-liver radioactivity ratio and provides a quantitative diagnostic index of great significance for portal vein disconnection and shunting but does not display structure and compensatory circulation $(8,9)$.

Transsplenic portal scintigraphy is advantageous in being able to show the structure and compensatory circulation of the portal vein, as well as in allowing diagnosis of compensatory circulation by hemodynamic dysfunctionwhich cannot be achieved rectally. Besides, transsplenic portal scintigraphy is also superior in that in produces only a tiny puncture, requires only a fine transfixion pin, requires a low amount of contrast medium $(0.5 \mathrm{~mL})$, and causes only minor injury to the spleen. Not many articles, however, have dealt with the introduction of this technique into the clinical diagnosis of cirrhotic portal hypertension. 
This study aimed to investigate the clinical significance and ability of transsplenic portal scintigraphy to provide a comprehensive, effective, convenient, and safe method of diagnosing portal hypertension.

\section{MATERIALS AND METHODS}

This study enrolled 50 patients with cirrhotic portal hypertension (41 men and 9 women; mean age, $53.1 \pm 18.2$ y) and 10 controls ( 6 men and 4 women; mean age, $45.2 \pm 12.5$ y). The cirrhotic portal hypertension had been diagnosed on the basis of clinical examination, liver-function examination, history, and the results of ultrasound, CT, and gastroscopy. According to the Child-Pugh classification, 15 patients with cirrhosis were Child A, 19 were Child B, and 16 were Child C. The Qilu Hospital ethics committee approved the study, and written consent was received from all participants.

Imaging was performed on a SPECT camera (Millennium VG, 3/8-in crystal; GE Healthcare) with a high-resolution, low-energy, parallel collimator. The camera detector was positioned in the anterior view over the patient's upper abdomen so that the field of view would include the heart, liver, and spleen. The patient fasted before the examination, and $740 \mathrm{MBq}$ of ${ }^{99 \mathrm{~m}} \mathrm{Tc}$-phytate in $0.5 \mathrm{~mL}$ were percutaneously injected into the splenic parenchyma under ultrasound guidance (Logiq 7; GE Healthcare) using a $3.5-\mathrm{mHz}$ probe and an intercostal approach. Continuous dynamic images were captured at 1 frame/s for 32 frames with a matrix of $64 \times 64$. From sequential dynamic images, an operator identified the heart-pool image, defined the region of interest using a light pen, and then copied this region of interest over the right lobe of the liver. Time-radioactivity curves were

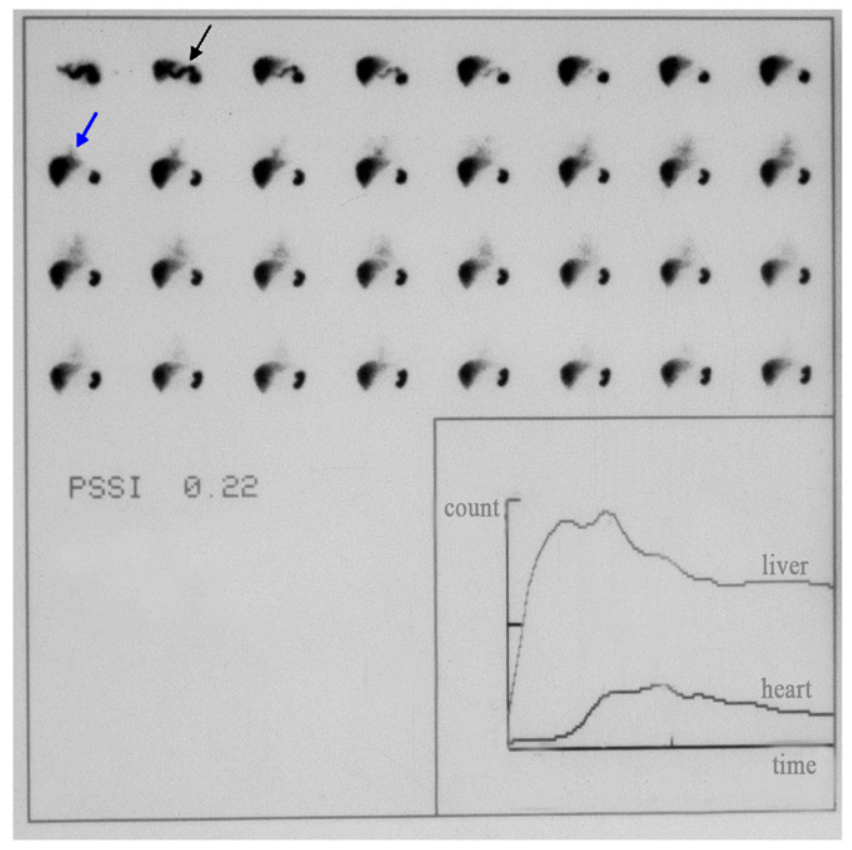

FIGURE 1. Continuous dynamic scintigraphy images were acquired at 1 frame/s for 32 frames with matrix of $64 \times 64$. Normal image of transsplenic portal scintigraphy in control group showed splenoportal vein shaped like letter S (black arrow). Heart-pool image appeared at $9 \mathrm{~s}$ (blue arrow). generated for the heart and liver. The portosystemic shunt index was calculated according to $\mathrm{H} /(\mathrm{H}+\mathrm{L})$, where $\mathrm{H}$ was the peak count of the heart time-radioactivity curve and $\mathrm{L}$ was the peak count of the liver time-radioactivity curve.

Ultrasound (Logiq 7) was used to detect the width of the portal and splenic veins. Gastroscopy (EG-2940; Pentax) was used to diagnose esophageal and fundal gastric varices, which were graded as mild, moderate, or severe.

Results were expressed as mean $\pm \mathrm{SD}$. The significance was evaluated by the Student $t$ test. Differences with probability values of less than 0.05 were considered significant.

\section{RESULTS}

\section{Transsplenic Portal Scintigraphy}

In the control group (Fig. 1), the splenoportal vein was shaped like the letter S, with regularity and clear streamlining, and the main branch of the hepatic portal vein could be observed at 2-4 s of imaging. Liver parenchyma was rapidly imaged, with a clear outline and well-distributed radioactivity, whereas the radioactivity in the spleen was limited to the injection spot, and the heart pool was not visualized till 5-6 s of imaging (portosystemic shunt index, $0.19 \pm 0.07$ ).

Images of the portal hypertension group showed 3 types of portosystemic shunts. The first was intrahepatic (Fig. 2), characterized as expansion of the splenoportal and intrahepatic portal veins, weak liver imaging, and mild to moderate heart-pool imaging. This type was found in 13 patients ( 7 class A patients, 5 class $\mathrm{B}$, and 1 class $\mathrm{C}$; portosystemic shunt index, $0.52 \pm 0.19)$. The second type

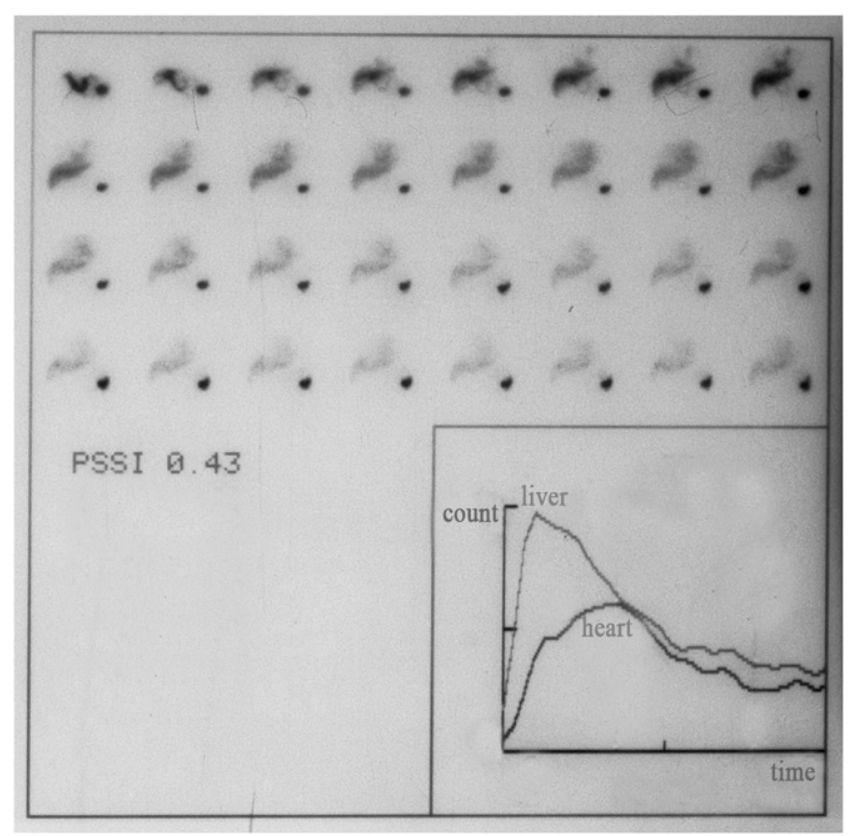

FIGURE 2. Sequential scintigraphy images acquired at 1 frame/s for 32 frames with matrix of $64 \times 64$. Intrahepatic shunt is characterized as expansion of splenoportal vein and intrahepatic portal vein, weak liver imaging, and mild to moderate heart-pool imaging. 


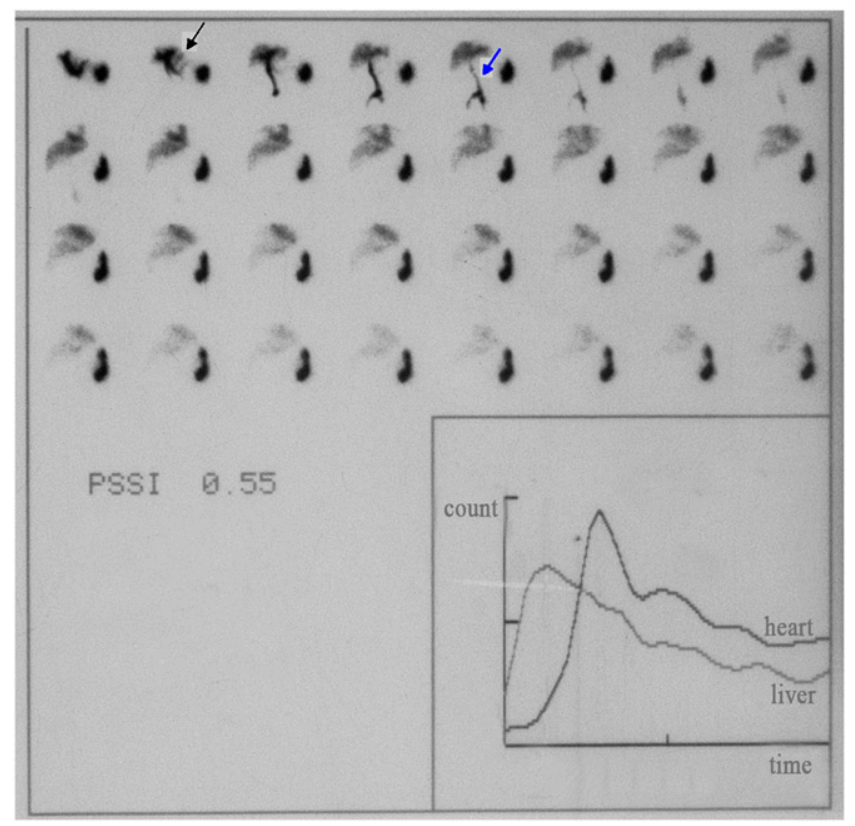

FIGURE 3. Sequential scintigraphy images acquired at 1 frame/s for 32 frames with matrix of $64 \times 64$. Compensatory shunt has uphill collateral branch (left gastric vein, black arrow) and predominant downhill collateral branch (inferior mesenteric vein, blue arrow).

was compensatory, characterized as an unclear or significantly expanded splenoportal vein, sparse liver imaging, partial spleen imaging, clear heart-pool imaging, and collateral vessels that might be predominantly uphill (left gastric vein), downhill (inferior mesenteric vein), or complex (Fig. 3 ). This type was found in 31 patients (8 class A patients, 13 class $\mathrm{B}$, and 10 class $\mathrm{C}$; portosystemic shunt index, $0.64 \pm$ 0.28). The third type was completely extrahepatic (Fig. 4), characterized as a highly expanded splenic vein, an unclear portal vein with a thick collateral branch, and a dense heart pool. This type was found in 6 patients ( 1 class B patient and 5 class $\mathrm{C}$; portosystemic shunt index, $0.91 \pm 0.03$ ).

\section{Correlations Among Parameters}

The portosystemic shunt index increased as cirrhosis worsened (Table 1), with significant differences among groups $(P<0.05$ or 0.01$)$. The width of the portal and

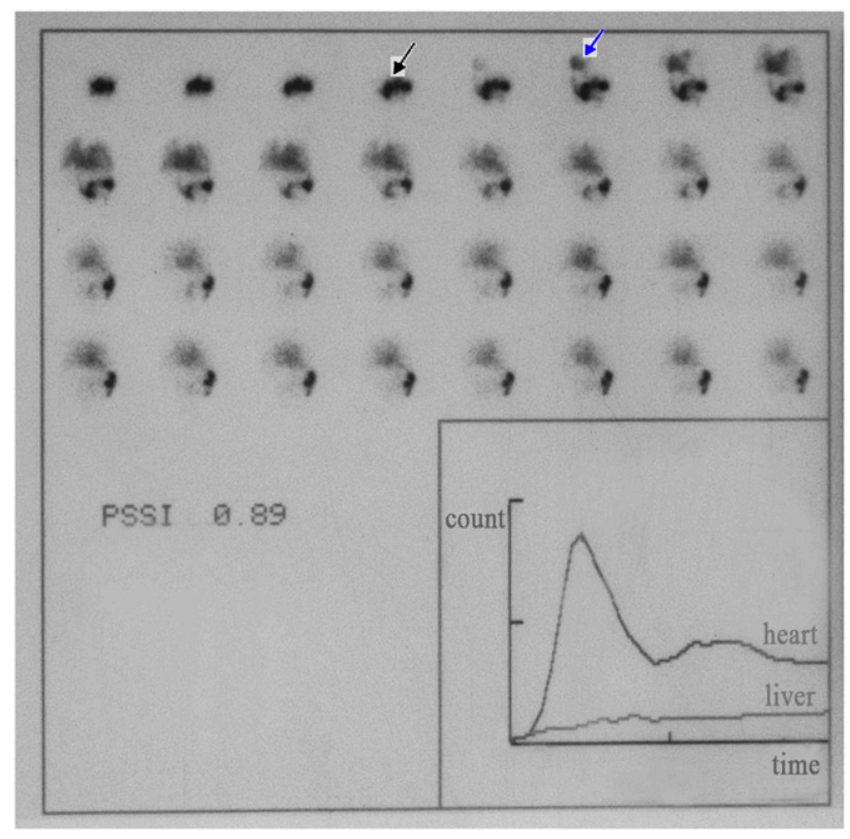

FIGURE 4. Sequential scintigraphy images acquired at 1 frame/s for 32 frames with matrix of $64 \times 64$. Completely extrahepatic shunt is characterized as highly expanded splenic vein (black arrow), unclear portal vein with thick collateral branch, and dense heart pool (blue arrow).

splenic veins also increased with Child-Pugh class, but with no significant differences among groups $(P>0.05)$.

The width of the portal vein increased, gradually, as the grade of gastric varices increased (Table 2). However, no significance was found $(P>0.05)$. The portosystemic shunt index also increased gradually, but with statistical significance between groups $(P<0.01)$.

\section{Value of Transsplenic Portal Scintigraphy in Predicting} Effect of Endoscopic Variceal Ligation

Among 31 patients with the compensatory type of shunt, the collateral vessels were predominantly uphill in 16 patients, downhill in 11 , and complex in 4 . The 20 patients with predominantly uphill or complex collateral vessels underwent endoscopic variceal ligation. Follow-up with gastroscopy for $1 \mathrm{y}$ found recurrence of the esophageal varices in 7 of the 16 patients with uphill collateral vessels

TABLE 1. Correlation Between Width of Portal Vein, Width of Splenic Vein, Portosystemic Shunt Index, and Different Child Classes of Cirrhosis Group

$\begin{array}{lcccc}\text { Child class } & \text { No. of cases } & \text { Width of portal vein }(\mathrm{cm}) & \text { Width of splenic vein }(\mathrm{cm}) & \text { Portosystemic shunt index } \\ \text { A } & 15 & 1.33 \pm 0.17 & 0.89 \pm 0.05 & 0.42 \pm 0.06 \\ \text { B } & 19 & 1.41 \pm 0.19 & 0.98 \pm 0.07 & 0.56 \pm 0.07^{\star} \\ \text { C } & 16 & 1.44 \pm 0.18 & 1.06 \pm 0.08 & 0.77 \pm 0.09{ }^{\dagger} \\ & & & \\ { }^{*} P<0.05 \text {. } & & & \\ { }^{\dagger} P<0.01 \text { vs. class A. } & & & \\ \text { Data are mean } \pm \text { SD. }\end{array}$


TABLE 2. Correlation Between Width of Portal Vein, Portosystemic Shunt Index, and Degree of Esophageal Varices

$\begin{array}{lccc}\text { Degree of varices } & \text { No. of cases } & \text { Width of portal vein }(\mathrm{cm}) & \text { Portosystemic shunt index } \\ \text { Mild } & 7 & 1.39 \pm 0.13 & 0.40 \pm 0.14 \\ \text { Moderate } & 12 & 1.44 \pm 0.17 & 0.58 \pm 0.18^{*} \\ \text { Severe } & 19 & 1.48 \pm 0.19 & 0.75 \pm 0.23^{*} \\ & & & \\ { }^{\star} P<0.01 \text { vs. grade } 1 . & & & \\ \text { Data are mean } \pm \text { SD. } & & & \end{array}$

and in only 1 of the 4 patients with complex collateral vessels. The portosystemic shunt index $(0.69 \pm 0.24)$ in the recurrence group was significantly higher than that $(0.61 \pm$ $0.19)$ in the nonrecurrence group $(P<0.05)$.

\section{DISCUSSION}

Portal hypertension is the primary cause of massive hemorrhage of the upper gastrointestinal tract and has many lethal complications. Thus, measuring the pressure in the portal vein is crucial for confirming therapy and predicting prognosis. It is generally held that portosystemic shunting increases as the pressure of the portal vein rises above a certain range. ${ }^{99 \mathrm{~m} T c-p h y t a t e}$ (radioactive colloid) injected into the splenic parenchyma passes through the splenoportal vein into the liver. Most of the colloid particles are captured by the Kupffer cells in the liver, and only a few enter the right heart, as explains the fact that the liver but not the heart is well imaged. Because of the elevated portal vein pressure and after portosystemic shunting, a portion of the colloid particles cannot enter the liver and instead enter the heart via compensatory circulation, which leads to simultaneous liver imaging and heart imaging. The radioactivity ratio between heart and liver reflects the extent of the portosystemic shunt and can be used to evaluate the extent of the elevation in portal vein pressure.

It is critical to maintain enough blood to the liver for prophylaxis of hemorrhage from rupture of esophageal varices (10). Varicosis occurred if the portal vein pressure was higher than $2.94 \mathrm{kPa}$, and rupture occurred if the portal vein pressure was higher than $3.43 \mathrm{kPa}$. As surgical therapy aims to control and prevent esophageal and fundal variceal bleeding, it is essential to estimate the liver function and portal hypertension of patients to determine perioperative management, and it is crucial to evaluate the anatomy of the portal system, compensatory circulation, and hemodynamics to select the type of operation $(11,12)$. A shunt is the first choice in the case of increased portal venous flow $(13,14)$, whereas disconnection may be the choice for those with decreased portal venous flow (15). Because of significant trauma and poor precision, many methods of monitoring portal venous pressure are not accepted by patients and are not widely used in clinical practice. Recent articles have reported that portal venous pressure can be precisely calculated by determination of heart and liver ratio with per rectum portal scintigraphy. The structure of the portal vein and compensatory circulation, however, cannot be displayed simultaneously $(8,9)$.

Our investigation showed that both the portosystemic shunt index and the structure of the portal vein and compensatory circulation could be determined by transsplenic portal scintigraphy. Knowledge of the flow direction of the compensatory circulation was essential in avoiding the omission of esophageal and supraesophageal branches due to disconnection and helped in determining the type of operation and whether nonoperative therapy was needed postoperatively. Our follow-up survey of endoscopic variceal ligation revealed that transsplenic portal scintigraphy can serve as guidance in predicting the result of endoscopic variceal ligation.

\section{CONCLUSION}

The splenic damage caused by transsplenic portal scintigraphy was mild, compared with that caused by radiography. No participants in this study reported malaise or complications. Transsplenic portal scintigraphy was safe and convenient, and it was sensitive in detecting the number and location of shunts, allowing improved surgical planning.

\section{REFERENCES}

1. Bosch J. Vascular deterioration in cirrhosis: the big picture. J Clin Gastroenterol. 2007;41(suppl 3):S247-S253

2. Szczepanik AB, Proniewski J, Huszcza S. Portal venous system after endoscopic sclerotherapy of esophageal varices in patients with liver cirrhosis: prospective study with Doppler sonography. Hepatogastroenterology. 2005;52:1448-1451.

3. Umeoka S, Koyama T, Togashi K, et al. Vascular dilatation in the pelvis: identification with CT and MR imaging. Radiographics. 2004;24:193-208.

4. Sheth S, Horton KM, Fishman EK. Vascular sequelae of cirrhosis: evaluation with dual-phase helical CT. Abdom Imaging. 2002;27:720-727.

5. Song B, Min P, Oudkerk M, et al. Cavernous transformation of the portal vein secondary to tumor thrombosis of hepatocellular carcinoma: spiral CT visualization of the collateral vessels. Abdom Imaging. 2000;25:385-393.

6. Kudo M, Zheng RQ, Kim SR, et al. Diagnostic accuracy of imaging for liver cirrhosis compared to histologically proven liver cirrhosis: a multicenter collaborative study. Intervirology. 2008;51(suppl 1):17-26.

7. Berzigotti A, Gilabert R, Abraldes JG, et al. Noninvasive prediction of clinically significant portal hypertension and esophageal varices in patients with compensated liver cirrhosis. Am J Gastroenterol. 2008;103:1159-1167.

8. Wang JY, Chen SL, Chen FZ, et al. A non-invasive method for evaluating cirrhotic portal hypertension by administration of ${ }^{99 \mathrm{~m}} \mathrm{Tc}-\mathrm{MIBI}$ per rectum. J Gastroenterol Hepatol. 1995;10:169-173.

9. Zhang Y, Qi G, Lui J. ${ }^{99 \mathrm{~m}}$ Tc-MIBI used for diagnosis of portal hypertension: an experimental study [in Chinese]. Zhonghua Wai Ke Za Zhi. 1995;33:754-756.

10. Cichoz-Lach H, Celiński K, Słomka M, et al. Pathophysiology of portal hypertension. J Physiol Pharmacol. 2008;59(suppl 2):231-238.

11. Chen W, Luo M, Sun YW, et al. Function of portal pressure during operation on the choice of surgical approaches in portal hypertension [in Chinese]. Zhonghua Wai Ke Za Zhi. 2008;46:1703-1711. 
12. Dragoteanu M, Balea IA, Dina LA, et al. Staging of portal hypertension and portosystemic shunts using dynamic nuclear medicine investigations. World $J$ Gastroenterol. 2008;14:3841-3848.

13. Wu ZY, Luo M. The relationship of hemodynamic changes in portal hypertension and the choice of surgical approaches in portosystemic shunt [in Chinese]. Zhonghua Wai Ke Za Zhi. 2008;46:1683-1685.
14. Ferral H, Patel NH. Selection criteria for patients undergoing transjugular intrahepatic portosystemic shunt procedures: current status. J Vasc Interv Radiol. 2005; $16: 449-455$.

15. Xu XB, Cai JX, Dong JH, He ZP, Han BL, Leng XS. Effects of portaazygous disconnection, portocaval shunt and selective shunts on experimental rat liver cirrhosis [in Chinese]. Zhonghua Gan Zang Bing Za Zhi. 2005;13:113-116. 